\title{
COMMENT
}

\section{Do all ecosystems maximise their distance with respect to thermodynamic equilibrium? A comment on the "Ecological Law of Thermodynamics" (ELT), proposed by Sven Erik Jørgensen}

\author{
RUTGER DE WIT \\ Centre National de la Recherche Scientifique and Université Montpellier II, \\ UMR 5119, Case 093, Place Eugène Bataillon, F-34095 Montpellier Cedex 05, France. \\ E-mail: rde-wit@univ-montp2.fr
}

This paper is dedicated to the late Professor Ramon Margalef, deceased 23 May 2004, who pioneered the study of the links between ecology, thermodynamics and information theory. The personal kindness of Ramon Margalef, and his stimulating and thought-provocative way of teaching and discussing issues in ecology and natural sciences have been a great source of inspiration.

SUMMARY: Sven Erik Jørgensen has boldly proposed a "Fourth Law of Thermodynamics" which he also advocates as the "Ecological Law of Thermodynamics" or ELT (Integration of Ecosystem Theories: A Pattern, Kluwer Academic Publisher). This Law is still a hypothesis and can be tested. I disagree with the generalised way he uses the term "exergy" in his approach. Exergy is commonly defined as a measure of the work capacity of a system. According to the physicists Boltzmann information embodies energy. Jørgensen takes this into account when considering the "exergy" stored in ecosystems; hence, according to his way of calculating the genetic information of the organisms is the mayor contribution to "exergy". I argue that energy has been embodied in the information during a historical process and that because of fundamental irreversibility this energy cannot be extracted again for work capacity. Moreover, other phenomena mentioned earlier by Ramon Margalef, demonstrate that proliferating information in biota shows uncoupling with respect to its embodied energy content according to the Boltzmann formula. In spite of these objections, some of Sven Erik Jørgensen's core ideas are exiting and worthwhile testing. I place these within the context of the non-equilibrium thermodynamic theories on "dissipative systems" developed by the physicist Ilya Prigogine.

Keywords: exergy, information, Gibb's free energy, embodied energy, genetic information.

RESUMEN: ¿MAXIMIZAN TODOS LOS ECOSISTEMAS SU DISTANCIA CON RESPECTO EL EQUILIBRIO TERMODINÁMICO? UN COMENTARIO SOBRE "LA LEY ECOLÓGICA DE LA TERMODINÁMICA". - De una forma muy marcada que llama nuestra atención, Sven Erik Jørgensen ha propuesto la "Cuarta Ley de la Termodinámica", que ha sido también promulgada varias veces como "Ley Ecológica de Termodinámica" (ELT), a ver Integration of Ecosystem Theories: A Pattern, Kluwer Academic Publisher. Hasta hoy, esta Ley es solamente una proposición, pero con la ventaja de representar una verdadera hipótesis con la característica que puede ser falsificada. Yo no estoy acuerdo con la manera generalizada como Jørgensen utiliza el término "exergy" para elaborar su teoría. La "exergy" de un sistema es normalmente definida como una medida de la capacidad de realizar trabajo por un sistema. El físico Boltzmann ha descrito claramente que la información engloba energía. Jørgensen utiliza este principio considerando la cantidad de "exergy" acumulada por el ecosistema. Así pues, Jørgensen considera que la información genética de los organismos que componen el ecosistema representaría la mayor parte de la "exergy" de este ecosistema. Mi opinión es que es absolutamente primordial considerar que esta información ha sido acumulada durante un proceso histórico y a causa de una irreversibilidad fundamental esta información no puede ser extraída otra vez para producir trabajo. Además, otros fenómenos que han sido mencionados en su tiempo por Ramon Margalef nos demuestran que la multipli- 
cación biológica de la información, que prolifera en los ecosistemas, tiene un gasto energético muy inferior de la energía acumulada por la información si es calculada según la formula de Boltzmann. Sin embargo, a pesar de estas objeciones, algunas de las ideas principales de Sven Erik Jørgensen, se colocan en el contexto de la teoría de la termodinámica fuera del equilibrio tal como ha sido elaborado por el físico Ilya Prigogine, las cuales son muy interesantes y merecen ser estudiadas para ser verificadas o falsificadas.

Palabras clave: “exergy”, información, energía libre de Gibbs, energía incorpodara, información genética.

If we divorce ourselves from biological knowledge and look at the biosphere as a mineralogist might see its contents, a number of the familiar concepts of natural history appear in a new light.

G. Evelyn Hutchinson

The Ecological Theory and the Evolutionary Play

Although living systems are extremely complex systems they obey the basic laws of physics. Hence, in ecological theory and ecological modelling it should be checked that each theoretical construct complies with the basic physical laws (Margalef, 1997), and models and hypotheses that violate these laws are refuted and should be abandoned. It is important to check this in an early phase of model development, because later it is much more difficult to detect flaws as the complexities of the model outcome and the real world obscure their comparison. A classical example is the formal application of the Lotka-Voltera equation for the predator-prey interaction resulting in fully reversible behaviour described as "pathological frictionless pendules" (May, 1973). However, while frictionless pendules are still possible under idealistic circumstances, i.e. in a complete vacuum, energy transfer in food chains always results in part of the energy being dissipated. The reversibility of the Lotka-Voltera equation violates the second law of thermodynamics, which dictates that all spontaneous natural processes create entropy and that therefore the use of energy for work is always less than $100 \%$ efficient. As a consequence of the second law of thermodynamics all natural processes have a "time-arrow" and all living systems, including ecosystems, possess an important historical component (Prigogine and Stengers, 1979).

Several authors have thought deeply about the implications of the three generally accepted laws of thermodynamics, i.e. the so-called First, Second and Third law of Thermodynamics, in order to understand the behaviour of living systems and of ecosystems in particular (Schrödinger, 1944; Margalef, 1968, 1980 and 1997; Prigogine and Stengers, 1979; Schneider and Kay, 1994; Ulanowicz, 1997; Jørgensen, 1997).
Sven Erik Jørgensen of the Royal Danish School of Pharmacy in Copenhagen has collaborated with colleagues worldwide on this subject and initiated the series "Ecosystems emerging" in the journal "Ecological Modelling" (e.g. Jørgensen et al., 2000). In general, the three laws of thermodynamics set the stage for system analyses and are particularly effective in describing system behaviour close to thermodynamic equilibrium. However, ecosystems are not in thermodynamic equilibrium at all and therefore it has been acknowledged that while the three basic laws remain valid there is some more basic physics beyond these three laws that is of uttermost importance for ecosystem functioning. The search is directed towards a difficult branch of science i.e. the so-called "Irreversible Thermodynamics" or more appropriately called "Non-Equilibrium Thermodynamics" (i.e. thermodynamics of non-equilibrium phenomena) an area initiated by Lars Onsager, Théophile De Donder and Ilya Prigogine in the 1930's which is still developing (Prigogine and Stengers, 1979; Prigogine and Kondepudi, 1999). To cope with the physics of ecosystems beyond the three Thermodynamic Laws, Sven Erik Jørgensen has been particularly thought provoking by boldly proposing a "Fourth Law of Thermodynamics" which he also advocates as the "Ecological Law of Thermodynamics", abbreviated as ELT, to highlight its claim of being particularly applicable to ecological systems.

The ideas behind the "Ecological Law of Thermodynamics" emerged in the seventies when Sven Erik Jørgensen worked together with Hennig F. Mejer (Jørgensen and Mejer, 1979). Despite using pretentious names such as the Fourth Law of Thermodynamics or ELT, Sven Erik Jørgensen has repeatedly pointed out that ELT is still a hypothesis and therefore he likes to add the adjective "tentative". A strong plea for the tentative ETL is proposed in his book entitled "Integration of Ecosystem Theories: A Pattern" of which I will discuss the second revised edition of 1997 (388 pp. Kluwer Academic Publisher, Dordrecht). On page 164 in this book the ETL is defined as "If a system has a throughflow of exergy, it will attempt to utilise the flow to increase its exergy (i.e. move further away 
from thermodynamic equilibrium); if more combinations and processes are offered to utilise the exergy flow, the organisation that is able to give the highest exergy under the prevailing circumstances will be selected". I will discuss several aspects of this proposal later, but at a first glance we can make three important observations: i) the ELT invokes thermodynamics of non-equilibrium phenomena and as such is defined as a phenomenological law, ii) it introduces the variable exergy, a concept that we will discuss very critically, and iii) the final part, by stating that the organisation that is able to give the highest exergy under the prevailing circumstances will be selected, offers a means to test the hypothesis.

The simplest case analysed by thermodynamics is a completely isolated system. In this case the second law predicts a continuous increase of entropy until a final stage of thermodynamic equilibrium of maximum entropy is achieved. Hence, thermodynamic equilibrium is the attractor for such a system (Prigogine and Stengers, 1979). Based on this principle life appears enigmatic. Or in other words, living matter evades decay to thermodynamic equilibrium. "How does this occur?" was the major question raised by a famous "naïve physicist" (Schrödinger, 1944). However, it is very important to realise that completely closed systems are rare in everyday life and all living systems, including organisms and ecosystems are non-isolated systems that exchange energy and entropy with their environment, and very often they are open systems that also exchange matter with their surroundings.

To illustrate the widespread occurrence of nonequilibrium phenomena in nature, I will discuss the simple case of an oxygen diffusion gradient that can be found everywhere in the sediments of aquatic systems. Oxygen from the water column diffuses down into the sediment until the depth where anoxic conditions occur and this mass transport is described by linear equations according to Fick's diffusion laws. Common language describes this process as "diffusion downslope driven by the concentration gradient" suggesting an analogy with a falling particle in a gravitational field that can be described by Newtonian mechanics using gravitation force. Nevertheless, such a Newtonian mechanical attracting force does not exist for the process of molecular diffusion, which can be explained merely by statistical phenomena applied to the behaviour of individual oxygen molecules. At temperatures above absolute zero, the oxygen molecules move randomly and in a collection of oxygen molecules move- ments in all directions can be observed at the submicroscopic level. By simply following statistical descriptions it can be shown that through a horizontal plane in the sediment more oxygen molecules will move downwards than upwards per unit time, because the population density (partial pressure) of oxygen molecules is higher above than below the plane. Thus, mass transfer is explained by basic statistics at the molecular level. A steady state diffusion gradient will be achieved if the sources and sinks remain constant over time. According to Prigogine, the steady state is the attractor for this system, which is characterised by a small amount of ordered structure (the concentration gradient) and a low rate of entropy production that is close to thermodynamic equilibrium (Prigogine and Stengers, 1979; Prigogine and Kondepudi, 1999).

Ilya Prigogine and co-workers have investigated cases where the system boundary conditions push the system further and further away from thermodynamic equilibrium. For example, they mathematically linked sources and sinks of selected chemical products with a series of chemical conversion reactions, and simulated behaviour both in a homogeneously stirred reactor as well as in a spatial diffusion field. They observed that by increasing input rates from sources and output from sinks above certain threshold rates, the original steady state solution for the system becomes unstable and new possibilities arise. The new possibilities combine a local increase of order with increased entropy production outside the local ordered structure. The flip-over to one of the novel possibilities can be achieved because fluctuations will disrupt an unstable steady state, and the new situation becomes reinforced by positive feedback loops. Hence, neither thermodynamic equilibrium, nor simple steady states continue to be the relevant attractors for these systems that are described as so-called dissipative structures (Prigogine and Kondepudi, 1999). The creation of dissipative structures is a historical process, combining stochastic and deterministic features and showing unexpected asymmetry. Nevertheless, these systems continue to obey the three Laws of Thermodynamics. Particularly the Second Law, since when considering the system and its environment the total sum of entropy is continuously increasing.

Ilya Prigogine's conclusion is that a strong dichotomy exists between systems that occur close to and far from thermodynamic equilibrium. The attractor for systems close to thermodynamic equilibrium is a steady state with little order. In contrast, systems far 
from thermodynamic equilibrium will evolve dissipative structures that become attracted further away from thermodynamic equilibrium and create a high degree of order locally (Prigogine and Kondepudi, 1999). Living organisms are striking examples of dissipative structures and seen this way, the many interacting organisms living in an ecosystem therefore comprise an even more complex dissipative structure together with their environment. Can ecosystems therefore be considered as dissipative structures sensu Prigogine? In this respect it is important to realise that all ecosystems have system boundaries that are open to energy and entropy transfer and in most cases also to matter transfer.

"Information" is an important concept in thermodynamics of system analyses. Most ecologists do not appreciate this aspect fully, because they use information theory restricted to describing biodiversity, a method that is rooted in communication theory. However, information can be used more broadly as a general measure for describing the existing limitation of possibilities (Margalef, 1968); it relates to order and thus to our capability to predict outcomes and reduce uncertainties and as such it is the opposite of entropy. Sometimes information is called negative entropy or negentropy in its contracted form. The link between order, information and statistics has its roots in the work of Boltzmann in the late $19^{\text {th }}$ century. Boltzmann showed that the macroscopic behaviour of complex physical systems can be described by a limited number of thermodynamic variables defined as a "macrostate". Within this "macrostate" "microstates" can be defined that describe systems at the molecular level. The entropy $(S)$ of a macrostate is thus defined by:

$$
S=k \ln \left(\Sigma \mathrm{p}_{\text {Microstates }}\right)
$$

Where, $\Sigma \mathrm{p}_{\text {Microstates }}$ represents the sum of the probabilities of all the microstates that give rise to the defined macrostate, and $k$ is a proportionality constant known as Boltzmann's constant, which is equal to $1.3803 \cdot 10^{-23} \mathrm{~J} /$ (molecules $\cdot$ degree). It is obvious that all the highly ordered life forms are microstates that are extremely improbable considering all the imaginable combinations of atoms and molecules on earth. Thus, the entropy of living systems is very low and the difference of this entropy with respect to thermodynamic equilibrium is considered negentropy or thermodynamic information carried by that particular life form. Figure 1 conceptualises the life forms and their capabilities to evade decay to ther-

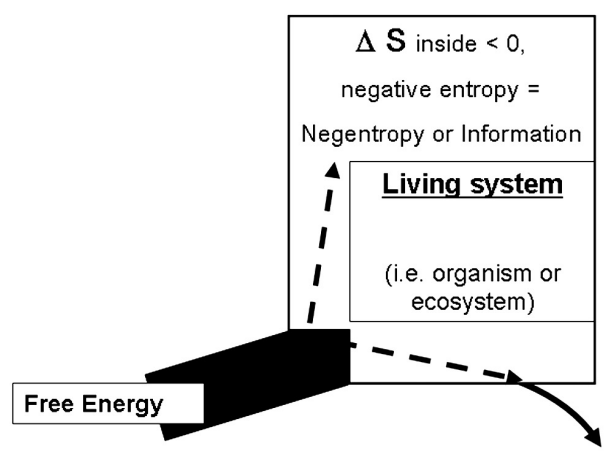

$\Delta S$ outside $>0$

$\Delta S$ outside $+\Delta S$ inside $>0$ (2nd Law of Thermodynamics)

FIG. 1. - Living systems as "dissipative structures" that thrive on input of free energy from outside. $\mathrm{S}=$ entropy. They combine a strong increase in entropy outside the system's boundaries $\left(\Delta \mathrm{S}_{\text {outide }}>0\right)$ with creation of order inside. $\Delta S_{\text {inside }}<0$, which can be described as negative entropy or information. However, the total entropy increases according to the second Law of Thermodynamics, i.e. $\Delta \mathrm{S}_{\text {outside }}+\Delta \mathrm{S}_{\text {inside }}>0$.

modynamic equilibrium as a dissipative structure.

Similarly, as Boltzmann has succeeded with physical systems to a certain level, Sven Erik Jørgensen tries to capture the overall behaviour of ecosystem functioning in a limited number of variables (cf. Boltzmann's macrostate) while considering that within this a great detail of specific cases and complexities can be described (cf. Boltzmann's microstates). Indeed, most ecologists restrain from making very detailed descriptions and modelling that are only completely meaningful for case studies, while some others look to unify concepts. We evaluate here if the approximations proposed by Jørgensen are meaningful in the last respect. In his framework, exergy is introduced as a key variable. Exergy has been defined as a measure of the work capacity of the system i.e. the amount of work that can be extracted from the system when it is brought into equilibrium with a reference system. Ideally, the reference system is the state of thermodynamic equilibrium. However, applied in the context of ecosystems there is some uncertainty of how to define the reference system. Typically it is assumed that this is an oxic environment and consequently it is calculated for an ecosystem assuming that all organic matter is oxidised to $\mathrm{CO}_{2}$, water and the most oxidised compounds like nitrate and sulfate for $\mathrm{N}$ and $\mathrm{S}$, respectively. Nevertheless, the original organic soup was probably anoxic and oxygenation of the atmosphere has been driven by life in the form of cyanobacteria and eukaryotic phototrophs. A first 


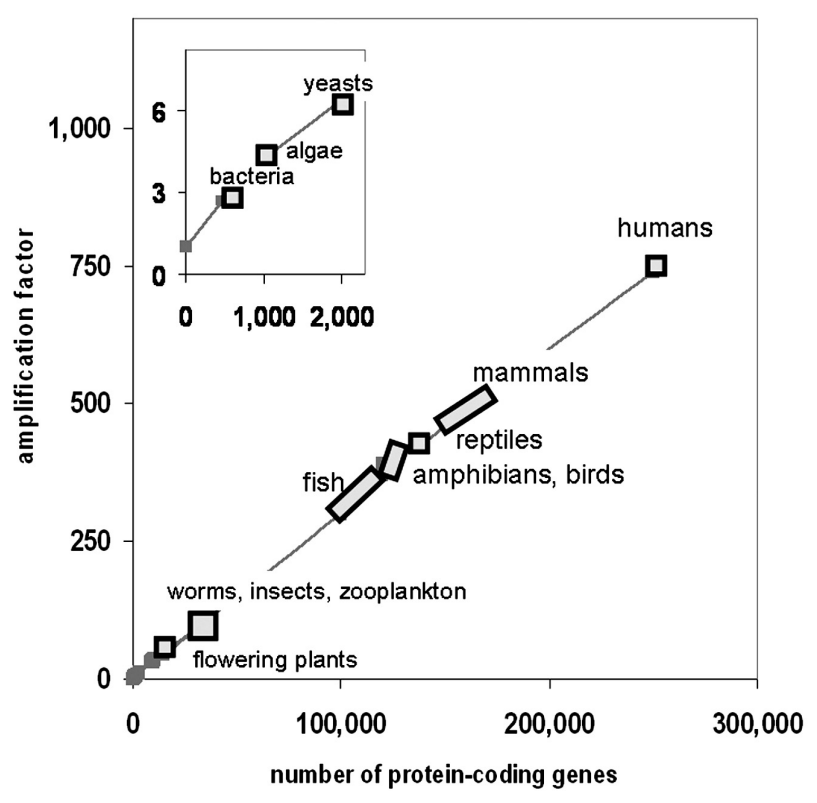

FIG. 2. - Relationship diagram for different life forms, concerning their number of protein coding genes and their amplification factor, used by Jørgensen ("Integration of Ecosystem Theories: A Pattern" second revised edition of 1997, 388 pp. Kluwer Academic Publisher, Dordrecht), to calculate the contribution to "exergy" storage in the ecosystem. The procedure consists of multiplying this amplification factor with the calorimetric free energy content of the corresponding biomass $(18.7 \mathrm{~kJ} / \mathrm{g})$. See text for explanation. Note the insert used for the lower values of the more primitive life forms.

difficulty for applying the exergy concept at the ecosystem level is thus the meaningful definition of the reference conditions. Exergy (Ex) is mathematically expressed as follows (e.g. Jørgensen, 1997):

$\mathrm{Ex}=S\left(T-T_{0}\right)-V\left(P-P_{0}\right)+\Sigma\left(\mu \mathrm{c}-\mu \mathrm{c}_{0}\right) \cdot N_{\mathrm{i}}$

Where, $S$ is entropy, $T$ absolute temperature, $P$ pressure, $\mu \mathrm{c}$ is the chemical potential for the different compounds and $N_{\mathrm{i}}$ is the mass of each compound expressed in moles. The index 0 indicates the value of the variable in the reference environment, and the non-indexed variables refer to the value for the ecosystem of interest. This definition of exergy is quite broadly accepted, although most ecologists are not familiar with it. In most cases we assume that the temperature and the pressure of both the ecosystem and the reference system are the same and do not change during work. For these conditions, it has been shown that the exergy expression becomes the same as the better known Gibbs free energy variable (e.g. Jørgensen, 1997).

Energy is conserved, but during each biochemical reaction the total entropy is increased and "energy loses quality" because a part of it is dissipated as heat. For the boundary conditions of constant tem- perature and pressure this is reflected by a destruction of the Gibbs free energy and therefore this variable is very practical in predicting the direction of biochemical reactions. Thus, a biochemical reaction can occur spontaneously if $\Delta \mathrm{G}$ is negative. A reaction with a positive $\Delta \mathrm{G}$ can only occur if it feeds on an external free energy supply (e.g. $\mathrm{CO}_{2}$ fixation driven by light energy). Jørgensen (1997), states that ecosystems that are open systems are dependent on throughflow of exergy; part of the exergy is dissipated in catabolic processes and part of it is stored in the ecosystem. Most ecologists would readily accept this statement if exergy were replaced by free energy.

Nonetheless, Sven Erik Jørgensen has created a lot of confusion among his colleagues by proposing his Ecological Law of Thermodynamics and claiming that ecosystems have a propensity to maximise exergy storage. I experienced this confusion myself and found it also expressed in the works of Schneider and Kay (1994), and Ulanowicz, (1997). I think the crucial issue creating the confusion is based on the fact that Jørgensen (1997), calculates the exergy content of living organisms by multiplying the calorimetric free energy content of their biomass (about $18.7 \mathrm{~kJ} / \mathrm{g}$ ) by a conversion factor that takes into account the number of so-called information genes and the number of cells. Figure 2 depicts the conversion factor proposed for different organisms in relation to the so-called information genes. Since this factor ranges from 3 for bacteria to 740 for humans it seems more appropriate to call this an amplification factor. Why should we multiply the calorimetric free energy content of the biomass by such high amplification factors to obtain a measure of their exergy?

The multiplication factors are based on information theory and the work of Boltzmann who related the information of a low probability event to a free energy content according to the formula:

$$
\begin{gathered}
\text { Free energy of information }= \\
=-R \cdot T \ln \left(\Sigma \mathrm{p}_{\text {Microstates }}\right)
\end{gathered}
$$

Where, $R$ is the gas constant, $T$ is the absolute temperature and $\Sigma \mathrm{p}_{\text {Microstates }}$ represents the sum of the probabilities of all the microstates that give rise to the event (cf. macrostate) see also Equation 1. In other words, order can be created at the expense of free energy and the amount of free energy needed is given by Equation 3 to achieve a given increase of information content. Information thus embodies energy. Jørgensen's approach can now be summarised as follows. The extremely low probability 
of an organism emerging from an oxidised abiotic "soup" environment is approximated by calculating the extremely low probability of obtaining a DNA sequence from random nucleotide sequences that is $100 \%$ aligned and identical with the major part of the genome of the organism. In this respect Jørgensen considers the protein-coding genes or open-reading frames. This probability is calculated and multiplied by the number of cells of the organism, because they all have the same DNA copies, to obtain $\left(\Sigma \mathrm{p}_{\text {Microstates }}\right)$. Hence the free energy content of information is calculated according to Equation 3, and the conversion factor per gram of corresponding biomass obtained. As a consequence, exergy in ecosystems is calculated by summing up the products of the genetic information and the biomass and thus most of the exergy is stored in the biocoenosis (the living part of the ecosystem). The value thus obtained is called "an exergy index" (Jørgensen et $a l ., 2000)$, and earlier it was simply called "exergy" (Jørgensen, 1997).

I think that this approach yields a very good estimator of the distance of ecosystems from thermodynamic equilibrium, but I find it very hard to make the link between this index and the original definition of exergy as a means of measuring a system's work capacity. I see no way of how the exergy amplification due to information storage can be liberated for work. Simply from a practical point of view, when predators eat prey they will use the free energy content of the prey biomass, but cannot extract free energy from the genetic information encoded in the genes. Genetic information is simply destroyed in these interactions, fortunately that is not so dramatic as it sounds, because while predators do not drive prey to extinction the persisting redundant information will allow the reestablishment of prey populations (Margalef, 1968). I recognise that the calculations of the "exergy index" (Jørgensen et al., 2000), or of "exergy" (Jørgensen, 1997), are consistent with a formal application of chemical potential as used in Equation 2, which includes a weighting of extremely low probabilities of finding complex organism when compared to the abiotic reference environment. Nevertheless, this index is absolutely useless for bioenergenetic calculations and unfortunately this is quite different from what is intended when suggesting that ecosystems are dependent on throughflow of exergy (see above). This is the root of the confusion.

I think that there is also a more formal flaw in the way that while information embodies energy according to the Boltzmann formula (Equation 3) we have to realise that this information has been embodied during a historical process and that it cannot be liberated again by a simple reversal of events which would be contradictory to the Second Law of Thermodynamics and the Time Arrow of Prigogine (Prigogine and Strengers, 1979). Behind Jørgensen's reasoning there seems to be an implicit generalisation of the main Gibbs formula: free energy = energy $-T \cdot S$ (where $T$ is absolute temperature and $S$ is entropy) reformulated by Herendeen as: energy - disorder $=$ energy + order (Herendeen, 1988, cited in Jørgensen et al., 2000), to mean exergy $=$ energy - disorder + order. Thus, I sincerely believe that the "order" term of this equation cannot be used as a food energy equivalent in ecosystems, which is quite contrary to Jørgensen's suggestion that ecosystems thrive on exergy throughput, while suggesting that exergy is partly dissipated and partly stored. I therefore recommend some rephrasing of concepts when discussing the hypothesis of the "Ecological Law of Thermodynamics", which in itself I find thrilling and worthwhile considering very carefully.

I suggest that the so-called "exergy index" sensu Jørgensen based on using amplification factors (see Fig. 2) be renamed as an "estimator of the distance from thermodynamic equilibrium" and that in bioenergetic calculations, including energy transfers in food chains in ecology, we stick to using free energy as a descriptor. For the latter, usually the Gibbs free energy can be used, which only needs to be replaced by another variable when biotic activity is closely related to pressure and/or temperature changes. Considering the former I think that using the term "estimator of the distance from thermodynamic equilibrium" is more appropriate when considering that it is still an imperfect measure of this distance as acknowledged by Sven Erik Jørgensen himself. Indeed, order as described by information measures is a very good estimator of distance from thermodynamic equilibrium. Ramon Margalef (1968), describes how ecosystem information is distributed in three channels, i.e. an ecological channel, a genetic channel and a cultural channel. Sven Erik Jørgensen chooses to focus on the genetic channel, a choice that seems understandable because the genetic channel has been tremendously enlarged during biological evolution (e.g. see Fig. 2). By focusing on the so-called information genes, which are the open reading frames or protein-coding genes, Jørgensen perhaps 
underestimates the information content of promotor regions and operon structures as well as the information content of ribosomic RNA coding genes. The latter have been used so elegantly during the last two decades to study the phylogenetic relationships among organisms. In addition, apparently superfluous DNA may operate in organisms and ecosystems as so-called "selfish genes" sensu Dawkins and should perhaps be included in the estimator. Therefore, at present, Jørgensen (1997, 2000), underestimates the genetic information content of organisms, but perhaps complete genome sequence analyses will shed some light on these issues. Often, the ecological information content is small compared to the genetic information (Margalef, 1968), and can thus often be neglected in this respect. However, for the specific purpose of analysing ecosystem growth, Ulanowicz (1997), uses the information content of the foodweb network in a meaningful way. When considering ecosystems with animals showing complicated ethological learning patterns and particularly when analysing ecosystems managed by humans and when we consider ecosystem engineering we should not underestimate the importance of the cultural channel.

Without explaining this aspect Sven Erik Jørgensen claims that his theory of maximizing distance from thermodynamic equilibrium automatically accommodates the Darwinian evolution theory. Referring to the many ideas of Ramon Margalef (1968; 1980; 1997), we can see that this is somewhat more complicated. First of all Margalef highlighted the importance of the historical process in ecology which links up with the ideas of Ilya Prigogine. He describes that the energy spent in creating a surviving species is extremely high because it must take into account the energy spent on the evolutionary game as a trial and error process, which therefore also includes the energy spent on phylogenetically related dead-ends. This energy expenditure is of course much higher than the embodied energy content of information calculated according to Boltzmann's formula. Surviving information has a higher historical embodied energy content than simple information. Ramon Margalef liked to compare this with the energy spent on developing a prototype of an industrial product. However, once the information is carried by a physical support system and copying and translating systems have been achieved in the historic process, copying information becomes a rather cheap process in terms of expenditure of free energy. Copying information often has a much lower energetic cost than the predicted embodied energy according to Boltzmann's formula (cf., Eq. 3). For biological systems Erwin Schrödinger postulated a role for non-periodic crystals as information carriers, which turned out to be the nucleotide sequence in the DNA with its double helix structure. Ramon Margalef's ideas were visionary and have proved pertinent, although currently no theoretical framework exists that can cope with the energy expenditure of developing the prototype (spent in the Darwinian evolutionary play) and the decreased energy expenditure in cheap copying. In conclusion, this highlights that proliferating information in biota shows uncoupling with respect to its embodied energy content according to Boltzmann's formula.

The link between energy expenditure and order in living systems is not straightforward and therefore the formula exergy $=$ energy - disorder + order cannot be applied to living systems. It is very important that the free energy throughput and the evolutionary and historically accumulated information are considered separately and should not be lumped together in an "exergy" term. Therefore, I propose to reformulate the basic concepts behind the "Ecological Law of Thermodynamics" as follows: "Ecosystems are open systems that depend on throughput of free energy. A part of the free energy is dissipated in catabolic processes, while ecosystems have a strong propensity to store information and thus increase their distance from thermodynamic equilibrium". The hypothesis itself can thus be reformulated as follows: If a system has a throughflow of free energy, in combination with the evolutionary and historically accumulated information, it will attempt to utilise the flow to move further away from thermodynamic equilibrium; if more combinations and processes are offered to utilise the free energy flow, the organisation that is able to give the greatest distance away from thermodynamic equilibrium under the prevailing circumstances will be selected" I find this hypothesis very interesting and worthwhile testing and I think that it links up nicely with the theory of dissipative systems proposed by Ilya Prigogine. Moreover, Sven Erik Jørgensen has provided us with some nice methodology, despite the fact that I reject his confusing way of using exergy as a unifying concept. The essential point is also that the hypothesis can be tested. Accordingly, for a given environmental setting one can imagine different biocoeno- 
sis. Sven Erik Jørgensen predicts that the biocoenosis that corresponds to the largest distance away from thermodynamic equilibrium will be selected. He claims that for about thirteen case studies it was indeed found that the prevailing biocoenosis was the one out of the considered possibilities that corresponded to the greatest distance from thermodynamic equilibrium in the environmental setting. How many cases need to be studied before we can generally accept such a phenomenological law based on empirical observations that support this hypothesis?

\section{Note added by the author}

Since submission of this comment, Sven Erik Jørgensen has published a new book on his theories together with Y.M. Svirezhev, which is entitled "Towards a Thermodynamic Theory for Ecological Systems", Elsevier Science and Technology Books (2004)

\section{REFERENCES:}

Jørgensen, S.E. and H. Mejer. - 1979. A holistic approach to ecological modelling. Ecol. Model., 7: 169-189.

Jørgensen, S.E. - 1997. Integration of Ecosystem Theories: A Pattern. Second revised edition. Kluwer Academic Publisher, Dordrecht.

Jørgensen, S.E., B.C. Patten and M. Straškraba. - 2000. Ecosystems emerging: 4. Growth. - Ecol. Model., 126: 249-284.

Margalef, R. - 1968. Perspectives in Ecological Theory. The University of Chicago Press, Chicago.

Margalef, R. - 1980. La Biosfera entre la termodinámica y el juego. Ediciones Omega, Barcelona.

Margalef, R. - 1997. Our biosphere Excellence in Ecology 10. Ecology Institute, Oldendorf/Luhe.

May, R.M. - 1973. Stability and complexity in model ecosystems. Princeton University Press, Princeton.

Prigogine, I. and I. Stengers. - 1979. La nouvelle alliance. Editions Gallimard, Paris.

Prigogine, I. and D. Kondepudi. - 1999. Thermodynamique, des moteurs thermiques aux structures dissipatives. Editions Odile Jacob, Paris.

Schneider, E.D. and J.J. Kay. - 1994. Life as a manifestation of the second law of thermodynamics. Math. Comput. Model., 19 (68): 25-48.

Schrödinger, E. - 1944. What is Life? Cambridge University Press, Cambridge.

Ulanowicz, R.E. - 1997. Ecology, the ascendent perspective. Colombia University Press, New York.

Scient. ed.: C. Marrasé 